\title{
Maturity Framework Enabling Organizational Digital Readiness
}

\author{
Carla G. MACHADO ${ }^{\mathrm{a}, 1}$, Peter ALMSTRÖM ${ }^{\mathrm{a}}$, Anna E. ÖBERG ${ }^{\mathrm{b}}$, Martin KURDVEE, \\ Sultan Y. ALMASHALAH ${ }^{\mathrm{a}}$ \\ ${ }^{a}$ Department of Technology Management and Economics, Chalmers University of \\ Technology, Gothenburg Sweden \\ ${ }^{\mathrm{b}}$ Volvo Construction Equipment, Operations Quality \& Safety, Arvika, Sweden \\ ${ }^{\mathrm{c}}$ RISE - Research Institute of Sweden, Materials \& Production Division, Mölndal, \\ Sweden
}

\begin{abstract}
Digitalization and the use of advanced technologies for more flexible and resource efficient production processes are changing the industrial competitive environment. However, new technologies are not seldom implemented without the whole organization being ready to fully utilize its benefits. This readiness can be expressed as organizational digital maturity. Previous studies identified that the level of digitalization is increasing, but, in many cases, due to lack of foundation, it is not aligned with the business strategy and/or supported by the organizational and technological infrastructures. There is a gap in existing models to provide practical starting-up steps to support the organizational digital readiness. A conceptual readiness framework for organizational digital readiness was tested through the analysis and reflection of four cases implemented by a Swedish manufacturing company. Findings point out that it is important to create a proper organizational foundation, a readiness, to ensure a company to evolve in digital maturity. That can be developed based on three main stages of change management practices. The tool can be used to identify the organizational readiness and thereby the gaps and thresholds necessary to overcome. This will enable companies to utilize the technology level necessary to increase cost and resource efficiency, and hence competitiveness. In addition, dimensions and a roadmap for organizational, digital, and smart readiness is presented.
\end{abstract}

Keywords. Organizational readiness, digital transformation, maturity, digitalization, Industry 4.0 .

\section{Introduction}

The Swedish industry is facing challenges moving towards digital transformation (DT). Previous studies found that most of these challenges are similar to industries all around the world, e.g. integration of the organizational structure, lack of knowledge and skills, competitive pressures, among others $[1,2]$.

Besides many definitions of DT this research considers it to be the combination of effects of different digital technologies and innovations leading to a radical organizational change, where norms, ways of working and structures are challenged. The norms and values in the company requires a new level of legitimacy to interact with the

\footnotetext{
${ }^{1}$ Corresponding Author. gcarla@chalmers.se
} 
new digital ecosystem [3, 4]. De Sousa Jabbour et al. [5] identified readiness for organizational change as one of the critical factors to ensure success and competitiveness in Industry 4.0, continually re-shaping and adapting the structure, goals, and strategy.

According to Lokuge et al. [6] and Kane et al. [7] companies are struggling to achieve DT because of the lack of digital organizational readiness, i.e. the level of preparation and willingness to change for adoption, assimilation, and exploitation of digital technologies. Companies, in general, are focusing on technological matters forgetting to start with the right questions: What do we want to achieve? How can our competitiveness benefit from digitalization? What changes needs to be in place? [2]

Weiner [8] state that readiness for change is a construct formed by the relationship between structural and psychological factors, i.e. people involved feel committed to implementing the change, and believe that they have capacity to do it. The sense of readiness is generated by: definition of value(s) (needs and benefits of the change) and collective engagement around it; commitment of leadership (communication and actions); sharing information and experiences; organizational process for talent management; organizational culture; situational analysis (task demands, resources availability, time, etc.); and, organizational policies and procedures.

This research is part of a broader project, called SMART PM, focused on preparing companies for the digital transformation, and how to move up in terms of digitalization matureness. The main goal is to compile a self-assessment instrument, used by the companies themselves, that provide guidance on defining appropriated performance measures regarding digitalization and automation.

On this matter, two conceptual frameworks are compiled as result of previous studies $[2,9,10]$ presenting two approaches of assessment: organizational digital readiness and organizational digital maturity. According to Adom et al. [14, p.7], a conceptual framework is a structure developed to explain the progress of a studied phenomenon and its links with concepts, empirical research, and theories to systematize the knowledge.

Schumacher et al. [11, p.162] state that readiness assessment is applied "(...) before engaging in the maturing process whereas maturity assessment for capturing as-it-is state whilst the maturing process". Initial studies indicated that digital readiness/maturity models usually do not make this differentiation and there is a gap to provide practical starting-up steps to support the organizational digital readiness [2]. In this sense two research question arises: (1) what are the minimum requirements to enable organizational digital readiness? (2) what is the roadmap to improve organizational digital maturity towards digital transformation?

The frameworks presented in this paper are supported by empirical evidence collected from digitalization projects conducted by a Swedish manufacturing company during the last two years, interviews, case studies and workshops, and test of an existing self-assessment model, all realized within companies in the project, and presented in earlier publications $[2,10]$.

\section{Conceptual Background}

Newman [16, p. 4] defend the use of the term DT because "(...) transformation impacts the whole business (...) challenging everyone in the organization (...)”. Kane et al. [7] state that is necessary to develop digital capabilities capable of promoting integration and alignment between the organizational goals with processes, people, culture, and 
structure. Therefore, designing a path for organizational readiness for DT starts by evaluating company's own digital situation and capabilities, identifying where technological and organizational changes are needed [7, 17, 18].

Complementing, Sousa Jabbour [5] identified critical factors for implementing sustainability-oriented Industry 4.0 in a more sustainable way: management leadership, readiness for organizational change, top management commitment, strategic alignment, training and capacity building, empowerment, teamwork and implementation leading team, culture, communication, project management, and national and local culture.

Machado et al. [2, 10] found that organization and governance is a common dimension evaluated by the existing models, however, these are not offering a comprehensive scope for organizational digital readiness, e.g. the "IMPULS-Industrie 4.0 Readiness" [19]. Most challenges reported by companies are related to organizational aspects, which are even more challenging for smaller companies when starting the process for digital transformation.

Machado et al. $[2,10]$ identified that DT in manufacturing is, simultaneously, a topdown approach driven by values and a bottom-up approach driven by the operations intelligence. Therefore, to evaluate a company's evolution towards DT in the manufacturing industry, it is necessary to include technological and operational aspects, for both: readiness and maturity assessment. In this sense complementary dimensions (presence in at least $70 \%$ of the sample analysed) are: 'Digital Strategy \& Business Model', 'Connectivity \& IT Systems Architecture', 'Data Collection \& Analytics' and 'Machine \& System/Technology integration'.

Machado et al. [2] pointed that in its structure the sample of digital maturity frameworks analysed vary from 3 to 6 stages, and in the last case, five to 6 stages are grouped in three main stages with two transition stages between them, e.g. the 'IMPULS' readiness model [19]. From the set of twenty-one models analysed only six of them were developed as self-assessment tools, while the other ones were usually connected with consultancy services attached to its conduction. Gaps identified includes lack of startingup process, to check if the company has the minimum requirements implemented to start the process; fragmentation of the organizational aspects; and no practical guidelines.

Weiner [8] states that implementation effectiveness relies on the consistency and quality of the people involved in the initial phases of the process. Where organizational readiness is higher the people involved are more skilled and persistent, however, in situations of lower or non-existing organizational readiness there are more resistance and less efforts in implementing it.

Bititci et al. [15] found correlations between management style and maturity levels, where closed and controlled management were linked with lower maturity, on the other side, open and empowered management styles was linked with higher maturity.

A previous study pointed out that some companies, most large ones, are using a management structure for DT similar to a network [2]. This type of organizational structure is like the one suggested by John Kotter [20] called a dual operating system. Two structures working together, one represented by traditional hierarchical structure linked to a second one in a network structure leaded by a team, formed by different functional silos and hierarchical levels, to enable information flow, freedom and accelerated speed of change. Complementing, Parnaby and Towill [21, p.24) state that when "managing-by-projects" the "(...) managers have to learn how to lead an organisational structure with very natural group teams, both transient and steady-state operational teams". 
John Kotter developed two versions of a model for changing management [20, 22]. The first [22] intends to present steps in a sequential way with a small group driving the change, and the second one [20] with steps running concurrently and continuously, is led by a large group connected in a network, constantly seeking for opportunities and initiatives. Creating an analogy that fits with earlier results in the project, the leading model [22] can be related to the context of a small company and how they are organizing themselves for digital transformation, and the second one, about accelerating the change [22], reflects how larger companies are conducting it, as they need to engage a larger number of people. Steps of both models are listed in Table 1.

Table 1. Leading change versus accelerating the change [22, 20]

\begin{tabular}{ll}
\hline \multicolumn{1}{c}{ Leading } & \multicolumn{1}{c}{ Accelerating } \\
\hline Establish a sense of urgency & Create a sense of urgency \\
Create a guiding coalition & Build a guiding coalition \\
Develop a vision and strategy & Form a strategic vision and initiatives \\
Communicate the vision & Enlist a volunteer army \\
Empower action & Enable action by removing barriers \\
Generate short-term wins (recognition) & Generate short-term wins (business results) \\
Consolidating Gains and Producing More Change & Sustain acceleration \\
Anchoring New Approaches in the Culture & Institute change \\
\hline
\end{tabular}

Appelbaum et al. [23] describe the leading change model [22] as a 'rigid' approach, but still can be considered as pivotal reference and a good starting point for change. Pollack and Pollack [24] conclude that, in practice, the change process is more complex than Kotter's sequence of steps. It may have multiple instances or processes with overlapping stages in different paces of change, that need to be supported by a guiding team providing strategic alignment, more aligned with the second model [20].

Organizational readiness for change is a competence required for successfully implementing digital transformation. From the context of this research, Kotter's models can be a good reference for a conceptual framework for organizational digital readiness. The models complement each other, and its differences rely in the intensity and scope of the change.

\section{Research Design}

The research project, which this study is part of, follows the 'model of knowledge creation through interactive research' developed by Ellström [25], involving the research and the practical systems working in a collaborative way. These systems are driven by problems originated from research or practice and they are interlocked and cyclical, where collective learning cycles developed by researchers and the companies produce successive versions of conceptualizations.

The development of the conceptual frameworks is supported by results of previous studies, both guided by Becker's procedure [26] for developing maturity frameworks: systematic literature research and review of existing models [2,10], expert interviews [2], conceptual modelling and validation, and test in the field. In this phase, only the first framework, the organizational digital readiness, was tested regarding: feasibility (can it be followed?), usability (how easy it is to follow it?) and utility (does the framework provide a useful step towards solving the problem it is meant to solve?). Four cases were used to test the framework, described in Section 4.

The cases did not have digitalisation per se as their main purpose, but rather different kinds of operational improvements where digital technologies were solutions to the 
operational problems, see Table 2. Each project is treated as one case which were evaluated using a structured interview protocol with 37 questions grouped in three blocks inspired by Kotter's models [20,22] (the questions are not presented here due to the length of the paper). In total five interviews were conducted by a company representative with deep knowledge in operational improvement and digitalisation, and complementary information were collected in a second step by one of the researchers of the project. The interviewed were project leaders or project owners for the four selected cases, asked to reflect on their experiences from running the projects, following the principles of reflective research $[25,27]$. The data was compiled and reviewed by the company representative and two other researchers. Following the interactive approach, results were presented to companies' experts for evaluation of its feasibility/usability/utility, and for improvement suggestions (structure of the framework and questions' clarity), resulting in an improved version of the questionnaire.

The conceptual digital maturity framework was presented for the companies within the project in a workshop, and results will be detailed in future publication, as the questions and ranking systems are still under development.

\section{Conceptual frameworks for organizational digital readiness and maturity}

This section presents two conceptual frameworks. They are considered complementary in the sense that companies can use the first one before initiating projects towards digital transformation and the second to evaluate ongoing projects.

\subsection{SMARTEM Readiness Framework}

This first framework contains the minimum requirements to be achieved to fulfil the first level of the maturity framework, the readiness level. The organizational readiness level represents the foundation that supports the evolution. However, during the interactive research phase it was verified that companies were adopting different strategies to startup the process towards digital transformation. All of them struggled to define the minimum requirements to improve the maturity path.

There is a gap in the existing models to provide an assessment of starting-up steps. This complementary framework is called SMARTEM, an acronym inspired by the project name "SMART PM - Sustainable Manufacturing by Automated Real-Time Performance Management" and by its principles: leaderShip, teaM, collAboRation, sTructure, and pErformance Management. The framework can be used as an assessment tool to identify gaps in companies that already have some digital initiatives but are facing organizational and governance challenges, or as a guideline tool for companies that are taking the first steps towards DT and is formed by three blocks:

a) Block 1: Creating a climate for the digital transformation: digital organizational readiness needs to be built on a solid ground with a clear direction and goals. The focus is to establish the reasons why the company needs becoming more digital, to identify the leading group that will guide the company through the change, and to develop the vision for the change focusing on a long-term perspective. Based on a thorough evaluation of the needs, opportunities and threats, risks, weakness, and strengths, and with clear strategic benefits defined, the team needs to ensure awareness and engagement of the top management and other stakeholders involved. The initiatives need to be used to increase awareness around the vision, 
to improve competences and to identify opportunities or barriers for integration (internal or cross-companies).

b) Block 2: Engaging and attracting people around the digital transformation: vision and strategies need to be communicated to create awareness, a sense of purpose and minimize resistance during the digital change process. Attracting people involves internal and external strategies to aggregate people, develop the necessary skills and competences for the project, identifying competence gaps and define strategic hiring or outsourcing process. It is important to empower new groups (volunteers from different areas) to speed the change ensuring the necessary resources (e.g. time, knowledge, investment capital). DT is lead and enabled by engaged people, not necessarily external. However, it is necessary to remove barriers (e.g. norms, or lack of skill) to provide freedom and capabilities for crossboundaries projects.

c) Block 3: Consolidating and sustaining the digital transformation: identifying the performance and results of the project is critical to demonstrate the benefits of the change and promote the credibility of the leading group. It is necessary to identify the constraints, necessary capabilities, and enablers for digital transformation. Results and lessons learned from the projects need to be organized and shared across the company, building the momentum, and ensuring continuity. The main challenge is to transform the tacit knowledge (from the experience) to explicit (e.g. creating new routines and ways of working, etc.). Results should be used to identify new leaders in the network.

The blocks represent a set of capabilities to position the company on a DT roadmap incorporated in a questionnaire and by a final five-points scale question to evaluate each block. The main goal on having questions and the five-point scale question is to compare the perception of the group involved and the processes developed during the initiatives, as sometimes there is a gap between perceptions and reality.

\subsection{SMART PM Digital Maturity Framework}

Maturity-based frameworks can be considered a practical way for organizing complex factors into capabilities and promote awareness. They can provide, a place to start, guidelines based on best practices, a common language and a shared vision, prioritization support, among others $[12,13]$.

To address gaps in the literature as well as improve practical applications, a framework has been developed based on a synthesis of empirics and best practices from the literature. The framework has been reviewed by the industrial stakeholders, driven by practical interest. The empirical experience was thus put into a theoretical context to improve the practical methods used [29].

The framework presents the core components of a maturity model identified by Lasrado et al. [28]: three main maturity stages with a set of distinct and testable characteristics (Table 2); dimensions formed by multi-dimensional factors with specific characteristics at each stage; "triggers" representing the specific conditions to satisfy in order to move to the next stage (these last two not detailed in this paper).

The three main levels and their roadmaps were developed by comparing existing models and case-studies, listed below in Table 2. They are not quantitative since they should not be used to grade or pressure the company, but as a supporting tool for assessment. 
As stated in Section 1, the dimensions in the framework represent technological and operational capabilities, implemented by its sub-dimensions covering company's process, organization, technology, and infrastructure, listed in Table 3 and its relationship illustrated on Figure 1.

Table 2. Maturity levels and roadmaps

\begin{tabular}{|c|c|}
\hline Maturity levels & Roadmap for maturity \\
\hline $\begin{array}{l}\text { Organizational maturity: The company has } \\
\text { developed the sufficient conditions, } \\
\text { organizational and technological, to support } \\
\text { thee digital transformation, which evolves from } \\
\text { creating awareness to institute a new culture. }\end{array}$ & $\begin{array}{l}\text { Identifying the needs and the urgency; defining the } \\
\text { leadership and the vision; promote awareness; empower } \\
\text { others; pilot projects and organize the wins and lessons; } \\
\text { sustain the moment and ensure continuity prioritizing } \\
\text { projects; and institute the culture. }\end{array}$ \\
\hline $\begin{array}{l}\text { Digital maturity: The company is digitally } \\
\text { connected and has developed the necessary } \\
\text { infrastructure and processes to integrate the } \\
\text { cyber-physical systems }\end{array}$ & $\begin{array}{l}\text { Define a digital agenda; digital strategy and monitoring; } \\
\text { increase level of collaboration; expand the view of } \\
\text { transformation: horizontal and vertical integration; fully } \\
\text { digitized; capabilities increased cross-areas; expanded } \\
\text { view of IT systems support. }\end{array}$ \\
\hline $\begin{array}{l}\text { Smart maturity: Production system fully } \\
\text { integrated, collaborative, and able to respond in } \\
\text { real time to new conditions and demands in } \\
\text { the factory, in the supply network and } \\
\text { concerning customers. }\end{array}$ & $\begin{array}{l}\text { Enterprise-wide innovation management; new business } \\
\text { models and portfolio; digital culture; transformation } \\
\text { updated dynamically; flexible IT architecture; } \\
\text { integration and interoperability; real-time data collection } \\
\text { and analytics; future scenarios simulation; lead the } \\
\text { business ecosystem. }\end{array}$ \\
\hline
\end{tabular}

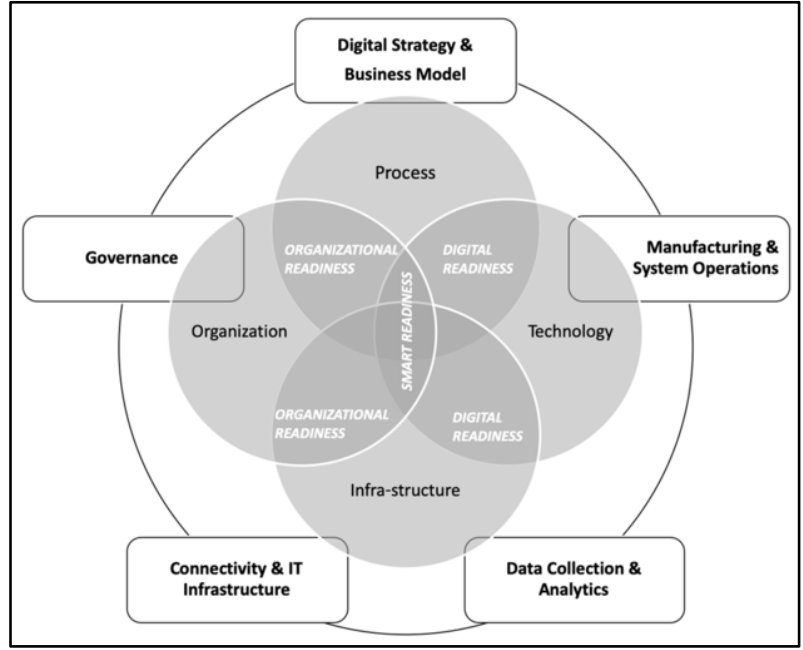

Figure 1. Digital maturity dimensions

Table 3. Dimensions and subdimensions

\begin{tabular}{ll}
\hline \multicolumn{1}{c}{ Dimensions } & \multicolumn{1}{c}{ Sub-dimensions } \\
\hline Organization \& Governance & $\begin{array}{l}\text { Cross-collaboration; investments; compliance; decision system; } \\
\text { leadership and culture; skills and competences; performance } \\
\text { management. }\end{array}$ \\
\hline Digital Strategy \& Business Model & Digital strategy; digital ecosystem; product development. \\
\hline Connectivity \& IT Architecture & $\begin{array}{l}\text { Connectivity; cyber-security; data processing; systems' } \\
\text { architecture. }\end{array}$ \\
\hline Manufacturing Systems \& & $\begin{array}{l}\text { Resource-efficiency; vertical and horizontal integration; } \\
\text { production; end-to-end engineering; and people. }\end{array}$ \\
\hline Dechnology & Digital twin; big data; data management and governance \\
\hline
\end{tabular}


To Lichtblau et al. [19] obstacles in the first level are similar to companies, e.g. little or no knowledge about digitalization and its benefits or threats, little or no market needs, very few or no skilled staff, and lack of corporate culture. In the highest stages can obstacles be lack of financial resources, norms, standards, legal frameworks, or skills. Overcoming these obstacles can be supported by assessing the minimum requirements using the SMARTEM framework, creating conditions to achieve organizational maturity. Maturity in this sense implies growing in capabilities and attitudes, indicating companies' consistency and commitment in projects, and implying that new processes and technologies are institutionalized $[5,13]$.

\section{Results from the cases}

The company site has around 1000 employees and started to discuss digitalization at the management level years ago, but in the last two years it became part of the strategic agenda for the site. A smart team, an internal team of voluntary participants, was formed to support the initiative. The team included people from manufacturing engineering, fabrication, maintenance, assembly, IT, quality, and project management.

During the last year, a global, companywide, digitalization strategy was created. The company considers it under development because they see themselves as "(...) not mature enough to define the requirements yet". A core team was formed responsible to implement and coordinate similar smart teams at all plants. The main purpose of the smart teams is to define the vision and digital strategy, promote awareness and education, identify competences, challenges, and opportunities, and develop use-cases. The cases, described in Table 4, were initiated before the global strategy was launched.

A summary of the result provided by the questionnaire is available in Table 5. During the discussions it became clear that there are gaps in developing a broader description with internal strengths and weaknesses assessing company's digital current-state (e.g. facilities, equipment, competences, strategy, products, services, and systems) and also in identifying opportunities and threats of the technologies. Consequences can be seen in Cases 1 and 3, the first one is at standstill because responsibilities and software license costs were not previously defined and the second is on hold due to systems constrains from the supplier.

Cases 2 and 4 has a clear vision defined and somehow communicated. In the discussion, this was linked with more maturity in Block 2. Block 3 indicates a discrepancy in the results and the perception in Case 1. The discussion pointed at that because the results, lessons learned and performance measures are being registered in academic documents (e.g. thesis, reports, articles) but in an isolated way, they have not been used to identify competence gaps, improve awareness or promote integration.

Results presented in Table 6 indicates a positive perception regarding the company's capability for engaging and attracting people around the digital transformation, which maybe is enhanced by the implementation of the global DT project in the second part of 2019. The new structure that has been implemented is like the Kotter's dual operating system [20]. Regarding the sequence of process and stages for starting-up the digital initiatives, the discussions indicate that the processes within the stages do need to occur in a coordinated way, however, the sequence of stages was considered a good roadmap and structure to assess, plan and implement the initiatives. 
Table 4. Cases' description

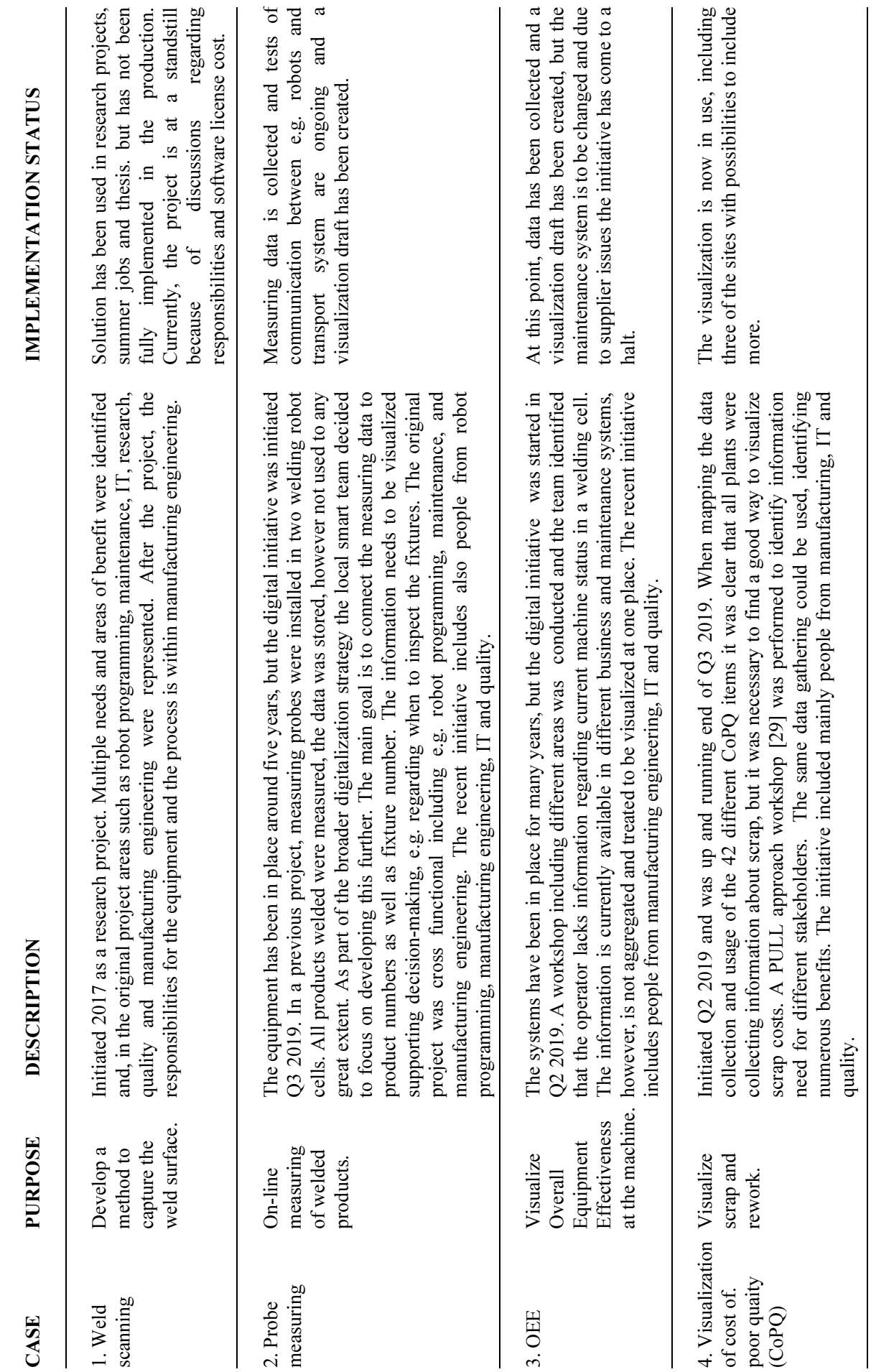


Table 5. Summary of the results from the interviews

\begin{tabular}{|c|c|c|c|c|c|}
\hline \multicolumn{2}{|c|}{ Requirements } & \multirow{2}{*}{$\begin{array}{c}\text { Case } 1 \\
(+)\end{array}$} & \multirow{2}{*}{$\begin{array}{c}\text { Case } 2 \\
(+)\end{array}$} & \multirow{2}{*}{$\frac{\text { Case } 3}{(+)}$} & \multirow{2}{*}{$\begin{array}{c}\text { Case 4 } \\
(++)\end{array}$} \\
\hline \multirow{12}{*}{1} & Opportunities & & & & \\
\hline & Threats & & & $(-)$ & \\
\hline & Needs & $(+)$ & $(+)$ & $(+)$ & $(++)$ \\
\hline & Risks & $(++)$ & $(-)$ & $(+)$ & \\
\hline & Strengths & $(+)$ & & $(+)$ & $(++)$ \\
\hline & Weakness & $(+)$ & & $(+)$ & $(++)$ \\
\hline & Strategic benefits & $(+)$ & $(+)$ & $(++)$ & $(+)$ \\
\hline & Visualization of the technical requirements & $(-)$ & $(++)$ & $(-)$ & $(-)$ \\
\hline & Clear vision & $(-)$ & $(++)$ & $(-)$ & $(-)$ \\
\hline & Communication of the vision & $(+)$ & $(+)$ & $(-)$ & $(-)$ \\
\hline & Awareness and engagement of the top management & $(-)$ & $(-)$ & $(-)$ & $(+)$ \\
\hline & Core team & $(-)$ & $(++)$ & $(+)$ & $(+)$ \\
\hline \multirow{3}{*}{2} & Digital awareness and/or trainings across company & $(-)$ & $(+)$ & $(+)$ & $(+)$ \\
\hline & Strategies to enroll and empower new people & $(-)$ & $(+)$ & $(-)$ & $(+)$ \\
\hline & Optimization and integration in the value chain & $(-)$ & $(+)$ & $(+)$ & $(+)$ \\
\hline \multirow{4}{*}{3} & Escalating digital benefits across the company & $(+)$ & $(+)$ & $(-)$ & $(+)$ \\
\hline & Results and lessons learned registered & $(+)$ & $(-)$ & $(-)$ & $(-)$ \\
\hline & Strategies to ensure the competence level & $(-)$ & $(-)$ & $(-)$ & $(-)$ \\
\hline & Performance measures for evaluate the digital initiative & $(+)$ & $(-)$ & $(+)$ & $(+)$ \\
\hline
\end{tabular}

Caption: (-) not identified; (+) Somehow identified; (++) Clear identified; (blanks) not answered

Table 6. Overall assessment

\begin{tabular}{|c|c|c|c|c|}
\hline & Case 1 & Case 2 & Case 3 & \\
\hline $\begin{array}{l}\text { Block 1: how structured and clear are the process of creating a } \\
\text { climate for digital transformation? }\end{array}$ & (1) & (2) & (2) & (2) \\
\hline $\begin{array}{l}\text { Block 2: how do you evaluate the processes for engaging and } \\
\text { attracting people? }\end{array}$ & (2) & (1) & (3) & (4) \\
\hline $\begin{array}{l}\text { Block 3: how do you evaluate the processes related for } \\
\text { tracking the progress of the digital initiative and to sustain and } \\
\text { formalize the changes towards the digital transformation? }\end{array}$ & (1) & (1) & (2) & (1) \\
\hline \multicolumn{5}{|c|}{$\begin{array}{l}\text { Caption: Block 1: (1) no structure or clear guidelines for DT in place; (2) some structure in place but no clear } \\
\text { guidelines in place. Block 2: (1)Digital initiatives are not being used to improve digital awareness or } \\
\text { integration within the value chain; (2)Digital initiatives are being used to improve digital awareness among } \\
\text { the group involved, however, does not support integration within the value chain; (3)Digital initiatives are } \\
\text { being used to improve digital awareness among the group involved, top management and key internal } \\
\text { customers related to digital vertical integration; (4)Digital initiatives are being used to improve digital } \\
\text { awareness across company, new people are involved in the initiatives, and the success of a digital initiative is } \\
\text { measured by its level of digital vertical integration and/or horizontal integration. Block 3: (1)Digital initiatives } \\
\text { are not documented, and results are not tracked and used to sustain the vision of the digital transformation; } \\
\text { (2)Digital initiatives are partly documented but results are not tracked and used to sustain and improve the } \\
\text { vision of the digital transformation. }\end{array}$} \\
\hline
\end{tabular}

Gaps were identified and list of recommendations for starting-up requirements is presented: (1) establishment of a core team (cross-function and levels) responsible to develop the vision and strategies for DT and to ensure alignment between different digital initiatives; (2) the digital initiatives should be chosen based on a thorough evaluation of needs, opportunities and threats, risks, weakness and strengths, and with clear strategic benefits defined; (3) the digital initiatives need to be implemented and managed by crossfunctional teams, enrolled and empowered by the core team; (4) all steps need to be documented and grouped by similar goals to become business cases and benchmarks to support other digital initiatives; (5) necessary competences and skills, as well as new governance instruments, need to be clearly identified, developed and shared across the company, (6) performance measures for each digital initiative and for the DT strategy needs to be in place and shared in the different levels. 
Improving these capabilities create a favourable environment to evolve in the organizational digital readiness. In this sense, the research is of great value for the companies since it will support their digital transformation to make it more efficient and effective.

\section{Conclusion}

Two conceptual frameworks for DT were developed. Starting-up requirements are presented in the SMARTEM framework, that has been tested and the conclusions from those initial tests are reported. The framework was considered applicable, useful, and feasible by the companies in initial discussions and assessments to improve organizational digital readiness and guide implementation of new digital initiatives across the company. Kotter's steps were considered relevant when grouped in the blocks.

The SMARTEM framework represent the minimum requirements of Level 1 in the maturity framework, that is presented in a summarized way as its content is still on development.

The practical contribution of this research relies on the fact that, in the next development phase, the self-assessment tools and method description will be available in a digital platform accessed by different companies. These will also be part of a workshop, since they should not be used stand-alone, but rather applied in conjunction with other norms and guidelines.

The theoretical contribution include development of two complementary assessment frameworks and the process of engaging, implementing and verification. It relies on the support to companies towards digital transformation. The purpose is to identify if the companies are ready to start the development or not, and if they are following their chosen roadmap. The assessment process should facilitate discussion, promote critical reflection, and improve managerial capabilities [15]. Next research steps already identified are to improve the SMARTEM framework tool through workshops, conduct tests in other companies inside and outside the project and finalize guidelines to support its implementation. In parallel, further development and tests of the levels of the maturity framework are ongoing within some of the companies.

\section{Acknowledgement}

SMART PM project (2018-01583) is granted by the Swedish Strategic Innovation Program Production 2030, financed by Vinnova. The support is gratefully acknowledged by the authors and special thanks are given to the representatives from the companies for their time and resources they have assigned in support of the research. This research is also supported by the Chalmers Production Area of Advance (AoA Production).

\section{References}

[1] Ministry of Enterprise and Innovation of Sweden, 2016, Smart Industry - a strategy for new industrialization for Sweden. [Online]. Available: https:/www.government.se/498615/contentassets/ 3be3b6421c034b038dae4a7ad75f2f54/nist_statsformat_160420_eng_webb.pdf 
[2] C.G. Machado et al., Industry 4.0 readiness in manufacturing companies: challenges and enablers towards increased digitalization, Procedia CIRP, vol 81, 2019, pp. 1113-1118.

[3] B. Hinnings et al., Digital innovation and transformation: an institutional perspective, Information and Organization, vol 28:1, 2018, pp.52-61.

[4] Y. Yamamoto and M. Bellgran, Four types of manufacturing process innovation and their managerial concerns, Procedia CIRP, vol 7, 2013, pp. 479-484.

[5] A.B.L. de Sousa Jabbour et al., When titans meet - Can industry 4.0 revolutionise the environmentallysustainable manufacturing wave? The role of critical success factors, Technological Forecasting \& Social Change, vol 132, 2018, pp. 18-25.

[6] S. Lokuge et al., Organizational readiness for digital innovation: development and empirical calibration of a construct, Information \& Management, vol 56, 2019, pp. 445-461

[7] C Kane et al., Coming of Age Digitally, MIT Sloan Management Review, 2018, June. [Online]. Available: https://sloanreview.mit.edu/projects/coming-of-age-digitally/

[8] B.J. Weiner, A theory of organizational readiness for change, Implementation Science, vol 4:7, 2009, 19.

[9] C.G. Machado et al., Production management and smart manufacturing from a systems perspective, Advances in Manufacturing Technology XXXII, vol 8:XXXII, 2018, pp. 329-334.

[10] C.G. Machado et al., Conditions for start-up of digital transformation: experiences from manufacturing industries. Working Paper. Chalmers, TME, 2019.

[11] A. Schumacher, S. Erol, and W. Sihn, A maturity model for assessing Industry 4.0 readiness and maturity of manufacturing enterprises, Procedia CIRP, vol 52, 2016, pp. 161-166.

[12] A.J.G. Silvius and R. A. Schipper, Maturity Model for Integrating Sustainability in Projects and Project Management. In: Proceedings of 24 IPMA World Congress, Istanbul, 2010.

[13] Software Engineering Institute, Capability Maturity Model Integration - CMMI® version 1.3. Software Engineering Institute, Pittsburgh, Pennsylvania, USA, 2010.

[14] D. Adom, E.K. Hussein and J.A. Agyem, Theoretical and Conceptual Framework: mandatory ingredients of a quality research, International Journal of Scientific Research, vol 7:1, 2018, pp. 6-7.

[15] U.S. Bititci et al., Value of maturity models in performance management, International Journal of Production Research, vol 53:10, 2015, pp. 3062-3085.

[16] M., Newman, 2017, Digital Maturity Model (DMM): A new tool to navigate the maze of digital transformation. [Online]. Available at: https:/www.tmforum.org/training-certification/navigatingdigital-transformation/

[17] H. Kagermann et al., 2013, Recommendations for Implementing the Strategic Initiative Industrie 4.0, [Online]. Available: https:/www.din.de/blob/76902/e8cac883f42bf28536e7e8165993flfd/ recommendations-for-implementing-industry-4-0-data.pdf.

[18] R. Geissbauer, J. Vedso and Stefan S. (2016), Industry 4.0: Building the digital enterprise - PWC 2016 Global Industry Survey. [Online]. Available at: https://www.pwc.com/gx/en/industries/industries4.0/landing-page/industry-4.0-building-your-digital-enterprise-april-2016.pdf

[19] K. Lichtblau, et al., Industrie 4.0-Readiness, Aachen, Köln, 2015. [Online]. Available: https://www.industrie40-readiness.de/?lang=en

[20] J.P. Kotter, Accelerate: building strategic agility for a faster-moving world, Harvard Business Press, Boston, 2014.

[21] J. Parnaby and D.R. Towill, Exploiting the concept of a manufacturing system part I: the relationship with process control, Journal of Manufacturing Technology Management, vol 20:7, 2010, pp. 915-932.

[22] J.P. Kotter, Leading Change, Harvard Business School, Boston, 1996.

[23] S.H. Appelbaum et al., Back to the future: revisiting Kotter's 1996 change model, Journal of Management Development, vol 31:8, pp. 764-782.

[24] J. Pollack and R. Pollack, Using Kotter's Eight Stage Process to manage an organisational change program: presentation and practice, Systemic Practice and Action Research, vol 28:1, 2015, pp. 51-56.

[25] P.E Ellström, Knowledge Creation Through Interaction Research: A Learning Perspective, In: HSS-07 Conference, Jönköping University, May 8-11, 2007.

[26] J. Becker et al., Developing maturity models for IT management - a procedure model and its application, Business and Information Systems Engineering, vol 1:3, 2009, pp. 213-222.

[27] M. Alvesson and K. Sköldberg, Reflexive methodology: new vistas for qualitative research, SAGE Publishing, $2^{\text {nd }}$ ed, 2009.

[28] L.A. Lasrado et al., A Set Theoretical Approach to Maturity Models: Guidelines and Demonstration, In: Thirty Seventh International Conference on Information Systems, ICIS 2016, Atlanta, pp. 1-20.

[29] A.E., Öberg et al., A pull approach to performance measurement systems design (2019). In: Proceedings of the 26th EurOMA Conferece: Operations adding value to Society, Helsinki, Finland, 17-19 June 2019. 